\title{
Smoking-associated DNA methylation changes: no smoke without fire
}

\author{
Zdenko Herceg*,1 \& Srikant Ambatipudi**,2 \\ ${ }^{1}$ Epigenetics Group, International Agency for Research on Cancer, Lyon, France \\ ${ }^{2}$ Achutha Menon Centre for Health Science Studies, Sree Chitra Tirunal Institute for Medical Sciences \& Technology, \\ Thiruvananthapuram, Kerala, India \\ *Author for correspondence: hercegz@iarc.fr \\ **Author for correspondence: asrikant@sctimst.ac.in
}

First draft submitted: 15 May 2019; Accepted for publication: 18 June 2019; Published online: 24 July 2019

Keywords: biomarkers $\bullet$ chronic disease $\bullet$ DNA methylation $\bullet$ mediators $\bullet$ smoking

Tobacco smoking is one of the leading preventable causes of early disability and death worldwide. Since the 1980s, although the prevalence of daily smoking has reduced, the number of smokers has steadily increased across the globe [1]. This has contributed to a marked rise in smoking-associated chronic diseases such as cancers, vascular and pulmonary diseases [1].

Given the intentional underreporting and/or recall bias associated with tobacco smoking, there is a need to identify biomarkers for better assessment of smoke exposure. Further, the utility of these biomarkers for monitoring the efficacy of preventive strategies and for assessing the risk of developing chronic diseases needs further investigation. In this regard, changes in DNA methylation, the covalent addition of a methyl group to the cytosine base in DNA [2], have shown promise not only as a biomarker of tobacco smoke exposure but also as mediators of chronic disease development.

\section{DNA methylation changes as biomarker of smoking}

A number of studies have found an association between exposure to tobacco smoke and DNA methylation changes in blood samples from adults [3,4], neonates and children [5,6]. In fact, smoking is considered as one of the most potent modifiers of DNA methylation [7]. This strong association across multiple studies has been utilized to assess the potential of DNA methylation as a biomarker of smoking intensity as well as the duration in adults [3] and in neonates born to mothers smoking during pregnancy [8].

Conventionally, breath carbon monoxide and cotinine are used as biomarkers of recent smoking, although they have limited application in assessing cumulative smoke exposure and to distinguish between never and former smokers because of their short half lives $(\sim 16 \mathrm{~h})$ [9]. On the other hand, DNA methylation changes provide a robust alternative to assess both smoking dosage and duration. The altered pattern of DNA methylation is seen, albeit with attenuation, even after two decades of smoking cessation [3,4]. This becomes interesting in light of the fact that the risk of cardiovascular disease increases even with low dosage of tobacco smoking and remains slightly higher compared with never smokers even after a decade of smoking cessation [10].

Most of the studies on alteration of DNA methylation in response to smoking were performed on whole blood which is not the target tissue and may be affected by the altered proportions of individual immune cell types due to smoking. Nonetheless, the DNA methylation changes strongly associated with smoking in whole blood (such as AHRR, CYP1A1 and CYP1B1) were also seen altered in buccal epithelial cells [11], lung [12] and adipose tissue [13]. This points to the robustness of DNA methylation based biomarker of tobacco smoking, showing validity across multiple tissue types and possible biological relevance of these DNA methylation changes. Indeed a combined score of smoking-associated DNA methylation sites (polyepigenetic score) from blood was recently used to assess smoking index and damage to the smoking exposed tissues such as gums and lungs [14].

DNA methylation changes form robust biomarkers of tobacco smoke exposure which may prove useful in stratifying individuals at high risk of developing chronic diseases associated with tobacco smoking and also to assess the success of cessation programs. Although, very few studies have looked at the causal role of smoking- 
associated DNA methylation changes in the development and progression of chronic diseases. Thus we are unsure if the smoking-associated DNA methylation changes will prove a useful biomarker of smoking associated chronic diseases.

\section{DNA methylation changes as mediators of smoking-related chronic disease development}

In addition to being utilized as biomarkers of tobacco smoke exposure, DNA methylation changes are also being studied as mediators of chronic disease development. In molecular genetics, a mediator is defined as a molecular event that is on the causal path between an exposure and an outcome variable [15]. DNA methylation changes are interesting candidates for mediating the tobacco smoking associated risk of chronic disease development because they are involved in pathways during embryo development [2], are mitotically heritable [2] and are reversible upon removal of tobacco smoke [3]. Most of the published articles have studied the mediatory role of DNA methylation changes linking tobacco smoking and lung cancer as both smoking and DNA methylation changes have been associated with lung cancer development [16] [BATTRAM T ET AL., unpublished data]. The first mediation analysis looking at the link between tobacco smoke, DNA methylation and lung cancer reported that AHRR and F2RL3 methylation events mediated $\sim 37 \%$ of the total effect of smoking on lung cancer odds [16]. Recent studies using causal inference methods such as Mendelian randomization in whole blood have revealed that the DNA methylation changes may not be a consequence of tobacco smoking [17] and methylation at tobacco-associated sites may not be causally implicated in lung cancer [BATTRAM T ET AL., UNPUBLISHED DATA]. Interestingly, a recent systemsepigenomics study has suggested that the inactivation of the AHR pathway, and not hypomethylation of $A H R R$, is a potential causal event in lung carcinogenesis [18]. This highlights the caution to be exercised while directly extrapolating findings from conventional mediation analysis, although the causal impact of DNA methylation changes on target tissue needs to be explored further. On the other hand, a recent in-vitro study highlighted the role of DNA methylation changes as the primary events leading to KRAS oncogene-driven lung cancer development [19]. Similarly, DNA methylation variation of F2RL3 affects platelet reactivity which may impact cardiovascular health adversely [CoRbin L ET AL., UnPUblished DATA]. Thus, there is inconsistency surrounding the role of DNA methylation changes as causal events during the development of smoking-associated chronic diseases.

Although the DNA methylation changes in response to tobacco smoke may not be causally linked to lung cancer development, their role in initiating the process and their role as drivers of lung carcinogenesis cannot be ruled out [20]. Further, the role of smoking-associated DNA methylation changes in chronic conditions like cardiovascular diseases is currently under investigation. Thus, a careful amalgamation of population-based large-scale studies using causal inference methods and systems level approach coupled with mechanistic studies is the need of the hour to understand the causal and mediatory role of DNA methylation changes in chronic disease development.

\section{Conclusion}

Since the first report, a number of studies have unequivocally reinstated the role of DNA methylation changes as biomarker of tobacco smoking. Because several studies aimed at investigating the causality of tobacco smokingassociated DNA methylation changes produced inconsistent results, the causal link between DNA methylation induced by tobacco smoking in cancer and other chronic diseases is being explored further. There is the need to refine the identified DNA methylation sites to objectively identify data lacking questionnaire-based information on tobacco smoking. As we move further, we face new challenges on identifying biomarkers of electronic cigarettes and smokeless tobacco. Further research aimed at testing the utility of tobacco-specific DNA methylation changes in cancer causation, biomarker discovery and risk stratification is warranted.

\section{Financial \& competing interests disclosure}

The work on DNA methylome changes associated with tobacco smoking and cancer carried out in Z Herceg's group at IARC was made possible by grants from the Institut National du Cancer (INCa, France), the EC FP7 Translational Cancer Research (TRANSCAN) Framework (EpiMark), INCA/Plan Cancer, the Fondation Association pour la Recherche contre le Cancer (ARC, France) and la Ligue contre le cancer (France) to Z Herceg. The authors have no other relevant affiliations or financial involvement with any organization or entity with a financial interest in or financial conflict with the subject matter or materials discussed in the manuscript apart from those disclosed.

No writing assistance was utilized in the production of this manuscript. 


\section{Disclaimer}

Where authors are identified as personnel of the International Agency for Research on Cancer/WHO, the authors alone are responsible for the views expressed in this article and they do not necessarily represent the decisions, policy or views of the International Agency for Research on Cancer/WHO.

\section{References}

1. $\mathrm{Ng} \mathrm{M}$, Freeman Mk, Fleming Td et al. Smoking prevalence and cigarette consumption in 187 countries, 1980-2012. JAMA 311(2), 183-192 (2014).

2. Herceg Z. Epigenetics and cancer: towards an evaluation of the impact of environmental and dietary factors. Mutagenesis 22(2), 91-103 (2007).

3. Joehanes R, Just Ac, Marioni Re et al. Epigenetic signatures of cigarette smoking. Circ. Cardiovasc. Genet. 9(5), 436-447 (2016).

4. Ambatipudi S, Cuenin C, Hernandez-Vargas $\mathrm{H}$ et al. Tobacco smoking-associated genome-wide DNA methylation changes in the EPIC study. Epigenomics 8(5), 599-618 (2016).

5. Joubert Br, Felix Jf, Yousefi P et al. DNA methylation in newborns and maternal smoking in pregnancy: genome-wide consortium meta-analysis. Am. J. Hum. Genet. 98(4), 680-696 (2016).

6. Richmond Rc, Simpkin Aj, Woodward G et al. Prenatal exposure to maternal smoking and offspring DNA methylation across the lifecourse: findings from the Avon Longitudinal Study of Parents and Children (ALSPAC). Hum. Mol. Genet. 24(8), 2201-2217 (2015).

7. Breitling Lp, Yang R, Korn B, Burwinkel B, Brenner H. Tobacco-smoking-related differential DNA methylation: $27 \mathrm{~K}$ discovery and replication. Am. J. Hum. Genet. 88(4), 450-457 (2011).

8. Reese Se, Zhao S, Wu Mc et al. DNA methylation score as a biomarker in newborns for sustained maternal smoking during pregnancy. Environ. Health Perspect. 125(4), 760-766 (2017).

9. Benowitz Nl. Clinical pharmacology of nicotine: implications for understanding, preventing, and treating tobacco addiction. Clin. Pharmacol. Ther. 83(4), 531-541 (2008).

10. How Tobacco Smoke Causes Disease: The Biology and Behavioral Basis for Smoking-Attributable Disease: A Report of the Surgeon General. US Department of Health and Human Services (2010). https://www.ncbi.nlm.nih.gov/books/NBK53017/

11. Teschendorff Ae, Yang Z, Wong A et al. Correlation of smoking-associated DNA methylation changes in buccal cells with DNA methylation changes in epithelial cancer. JAMA Oncol. 1(4), 476-485 (2015).

12. Bakulski Km, Dou J, Lin N, London Sj, Colacino Ja. DNA methylation signature of smoking in lung cancer is enriched for exposure signatures in newborn and adult blood. Sci. Rep. 9(1), 4576 (2019).

13. Tsai Pc, Glastonbury Ca, Eliot Mn et al. Smoking induces coordinated DNA methylation and gene expression changes in adipose tissue with consequences for metabolic health. Clin. Epigenetics 10(1), 126 (2018).

14. Sugden K, Hannon Ej, Arseneault L et al. Establishing a generalized polyepigenetic biomarker for tobacco smoking. Transl. Psychiatry 9(1), 92 (2019).

15. Richmond Rc, Hemani G, Tilling K, Davey Smith G, Relton Cl. Challenges and novel approaches for investigating molecular mediation. Hum. Mol. Genet. 25(R2), R149-R156 (2016).

16. Fasanelli F, Baglietto L, Ponzi E et al. Hypomethylation of smoking-related genes is associated with future lung cancer in four prospective cohorts. Nat. Commun. 610192 (2015).

17. Li S, Wong Em, Bui M et al. Causal effect of smoking on DNA methylation in peripheral blood: a twin and family study. Clin. Epigenetics 1018 (2018).

18. Chen Y, Widschwendter M, Teschendorff Ae. Systems-epigenomics inference of transcription factor activity implicates aryl-hydrocarbon-receptor inactivation as a key event in lung cancer development. Genome Biol. 18(1), 236 (2017).

19. Vaz M, Hwang Sy, Kagiampakis I et al. Chronic cigarette smoke-induced epigenomic changes precede sensitization of bronchial epithelial cells to single-step transformation by KRAS mutations. Cancer Cell 32(3), 360-376 (2017).

20. Herceg Z, Ghantous A, Wild Cp et al. Roadmap for investigating epigenome deregulation and environmental origins of cancer. Int. J. Cancer 142(5), 874-882 (2018). 
